\title{
Link Mining: A Computer Vision and Pattern Mining Approach
}

\author{
Seema Mishra \\ Robotics and AI Lab, CC-1 \\ Indian Institute of Information Technology \\ Allahabad, India
}

\author{
G. C. Nandi \\ Robotics and Al Lab, CC-1 \\ Indian Institute of Information Technology \\ Allahabad, India
}

\begin{abstract}
This work addresses the important problem of discovery and analysis of social networks and link between frequent people observed from surveillance video footage where large amount of video data is collected routinely. A computer vision approach has been applied for detecting and extracting people within a group using HAAR classifier. This technique allows us to recognizing people by doing similarity matching between training faces and unknown detected face image. Therefore it is required to obtain high resolution face images of people in order to extract intrinsic feature information of facial images used in detection of person's faces. We present a novel frequent pattern mining based approach in the domain of frequent person detection i.e. apriori to solve frequent association problem between social networks obtained from low level task of face recognition. Our approach is illustrated with promising results from a fully integrated camera system.
\end{abstract}

\section{General Terms}

Data Mining, Frequent Pattern Mining, Social Network Analysis, Dynamic Social Network Analysis

\section{Keywords}

Dynamic social network analysis, Link analysis and mining, , Data mining, Frequent pattern mining, Knowledge Discovery, Computer Vision, key frame extraction, key frame selection.

\section{INTRODUCTION}

The applications of dynamic network analysis(DNA) is proliferated in various fields such as analysis of groups, understanding their dynamic relations and predicting as well as analyzing link between them. With reference to this dynamism, it is also able to discover the behavior of the group and its individuals [1]. Traditional social network analysis are incorporated with several computational techniques like artificial intelligence, machine learning, data mining to develop empirical research on human behavior, groups and organizational behavior within links among them with varying level of uncertainties.

There are two level of analysis in DNA. Firstly, it focuses on relational data, i.e.; data about a link between group of people, events and locations, organizations. Identifying associations between these entities is a crucial part of unveiling different types of activities in order to discover knowledge about network. The second is the focus on dynamism of the relations; i.e., how these relations likely to change in the future and what are the interesting consequences of these changes in the system.
There are several modern means of communications, like email, chartroom, weblogs, and telephone [14]. Here, we discuss about the new emerging means of communication and interaction is computer vision. Since computer vision techniques are being used tremendously in many areas of surveillance systems, face recognition $[2,19]$, crowd tracking $[15,16]$, detecting, recognizing and analysis of activities [17, 18 ] and events.

More specifically, we are attempting to discover higher level understanding of group behavior and their frequent relations within the reference of social interaction and dynamic network analysis. Understanding the behavior of individual and group, social networking methods assuage the analysts to revealing hidden patterns and knowledge from social communication [28, 35]. This discovered knowledge can be useful in law enforcement, homeland security, organizations, and closed world environment.

With the objective of achieving knowledge about social interaction, we can imagine our problem centered with the critical aspects of dynamic network analysis. For example, first aspect is frequent relation or link of people appearing together. Second aspect is dynamism in a group as people are changing within it. To help law enforcement, intelligent agencies, organizations or surveillance system in this research we propose a framework, for automated frequent link analysis in terms of social network pattern. The framework includes two level tasks, low level and high level. The low level task integrates module, face recognition and high level task includes pattern mining for frequent groups.

Here, the face recognition module should include:

1. Persistently track video sequences and bifurcate into key frames.

2. Dynamic training data set after detecting faces from frames.

3. Detect and recognize faces unambiguously under challenging conditions. For this purpose, only high resolute images of faces are captured.

Advancement in computer vision techniques has made this low level task performance possible in efficient and robust manner even in occlusion.

The approach we espoused for discovering frequent persons grouped together oftentimes is based on pattern mining approach [25]. 
The target of this paper is to contribute as follows:

1. We addressed the problem of discovering frequent group within the new framework of interaction for link mining between social networks in, possibly closed world, surveillance environment.

2. Modeling a group which should have set of individuals who are divulging similar characteristics, i.e. some proximity measurement in space or some orientation.

3. Analyze the frequent persons appearing in group using frequent pattern mining approach.

Integration of paper is delimited as follows that describe the stages involved in the system in more details: In Section 2, we put a description of social network and its creation. In Section 3 , we discuss proposed approach in the context of social network data. Section 4, incorporates insinuation of algorithm and section 5 discuss data sets and implementation results yielded by fully integrated system. This paper ends with conclusion in section 6 .

\section{SOCIAL NETWORK}

Since network is structurally designed as consisting of nodes and edges indicate relationship. Social network analysis is used to understand the pattern of interaction caused by social dynamics and events $[8,9,10,11,12,13]$. One special kind of network is known as social network and has been studying for long time $[3,4,5,6]$. Modeling complex datasets in graph has been recognized powerful tool in various research domains like chemical domain, computer vision, image and object retrieval, and machine learning. In particular, Dehaspe et al. applied Inductive Logic Programming (ILP) to obtain frequent patterns in the toxicology evaluation problem.

Social network graph corresponds to $G=\langle V, E\rangle$ as we have in mathematical literature where $V$ is set of nodes and $E$ is set of edges. Each node is an individual in society and possesses a name and some identifying information. This information could be face images and signatures.

Suppose, social connection strength is between two persons, $i$ and $j$. To establish strength of tie between individuals $i$ and $j$ the following assumptions are required $[8,38]$.

1. Persons involved in interaction, $i_{1}, i_{2} \ldots i_{m}$ where $\mathrm{m}$ is variable should be indentified and recognized in positive manner.

2. The interaction is measured in a group.

3. The frequency with which the groups of people are seen in proximity is observed in appropriate manner over the period of time.

To accomplish these assumptions pragmatically, we set low level job with face recognition module. With this premise, the face recognition system opportunistically detects and recognizes faces $[19,26]$ from the captured images from the video sequences. Face recognition module compute the probabilistic values i.e. $P=\left[p_{1}, p_{2} \ldots P_{N}\right]$ where each value evaluates the probability that recognized face is corresponds to individual $\mathrm{i}$ in the original data base of faces where signature of all individuals are stored. But if new face is detected, it should be stored in database of faces with subsequent index.
The index of face in original data base becomes the signature of recognized face. Suppose three persons P, Q, R are recognized from the system then we have $i^{\prime}=\operatorname{argmax}_{i} p^{i}, j^{\prime}=\operatorname{argmax}_{j} q^{j}$ and $k^{\prime}=\operatorname{argmax}_{k} r^{k}$.

\begin{tabular}{|c|c|c|c|c|c|c|c|}
\hline G/P & pl & $\mathrm{p}^{2}$ & p3 & . & . & . & $\mathbf{P n}$ \\
\hline$\overline{g l}$ & 1 & 1 & 0 & 1 & 0 & 0 & 0 \\
\hline$\overline{g 2}$ & 0 & 1 & 1 & 1 & 0 & 1 & 1 \\
\hline g3 & 1 & 1 & 1 & 0 & 0 & 0 & 1 \\
\hline . & 1 & 1 & 1 & 0 & 11 & 0 & 0 \\
\hline . & 1 & 1 & 1 & 1 & 0 & 0 & 0 \\
\hline . & 0 & 0 & 0 & 1 & 1 & 1 & 1 \\
\hline . & 0 & 1 & 1 & 1 & 1 & 1 & 1 \\
\hline & 1 & 1 & 1 & 1 & 0 & 0 & 0 \\
\hline $\mathrm{gm}$ & 1 & 1 & 1 & 1 & 0 & 0 & 0 \\
\hline
\end{tabular}

Figure 1. Grouping of people

Groups are obtained from capturing images of persons form fixed camera views. Here 1 indicates that particular person is involved in the particular group. Faces are recognized from these images and are used to build a social network which helps to compute frequent group that follows strong link between people in frequent communication. These social interactions in a group form the basis for discovering frequent group mining.

Now we can establish a link between these individuals and observe frequency of appearing them together with the appropriate metrics. Detected faces appear in a group and as a consequence we obtain a transaction of groups (see in figure1) as $G=\left\{g_{1}, \ldots g_{m}\right\}$ where $g_{i} \subseteq G$ and here $p_{1}, p_{2} \ldots p_{n}$ are persons

\section{FREQUENT PAERSONS AND THEIR LINK}

The proposed model of frequent link is pictured in figure 2 . We have divided the model in several modules of key frames extraction, face recognition, frequent pattern mining in faces recognized

The aforementioned system is conceptualized as in following algorithm 1 .

Algorithm 1: Frequent person and link mining

Input: set of video sequence

Output: frequent persons

1. Given Video sequence V captured routinely

2. Divide $\mathrm{V}$ into frames $F=\left\{f_{i} \mid i=1,2, \ldots N\right\}$

3. Calculate Key frames $K F \subseteq F=\left\{k f_{j} \mid j=1,2, \ldots K\right\}$

4. Face recognition returns matrix given as in figure 1 .

5. Compute frequent persons appearing using Apriori approach 


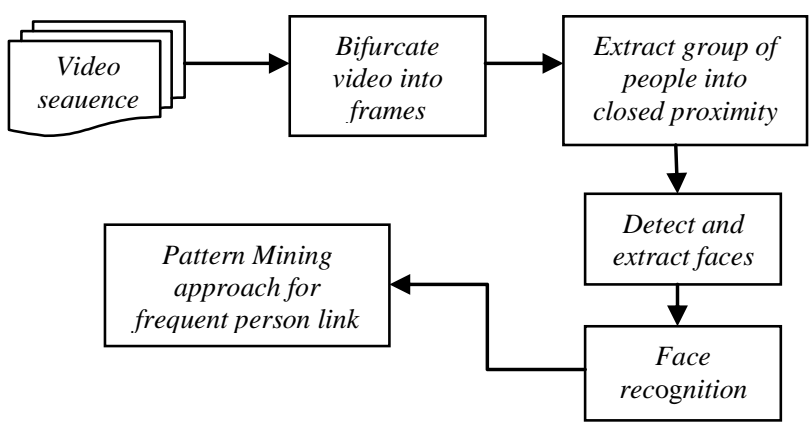

Figure 2: System for calculating frequent person and link mining

\subsection{Key Frames Extraction}

Our problem definition is concerned with the video capturing and fragment into several frames. This results to the very large database of images and in particular, much of the captured data may be redundant. Hence, it requires efficient method that enables to extract those frames which has desired information form video information to deal with called representative or key frames. The general idea of frames extraction is

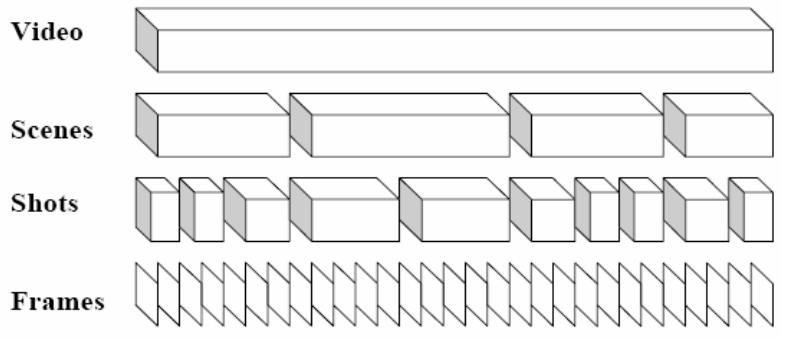

Figure 3

Here, our key frames to be extracted depend on the relevant object in visual contents which should consist of group of people.

We let video sequence $V d$ starting form time $t$ and comprising of $\eta_{F}$ frames is

$$
V d_{t}=\left\{f(t+n) \mid n=0,1, \ldots n_{F}-1\right\}
$$

and set of key frames $k f$ and $\eta_{K F} \leq \eta_{F}$ frames with the following expression

$k f_{i}=\left\{f_{K F}\left(t+n_{1}\right), f_{K F}\left(t+n_{2}\right), \ldots f_{K F}\left(t+n_{i}\right) \mid 0 \leq n_{i} \leq \eta_{F}\right\}$

Let $I(x, y)$ be the intensity value of each pixel in $m \times$ $n$ image at $(x, y)$ location. Our key frame selection process is based on the idea of reading image from left to right and calculate the gradient point where rapid change in intensity occurs. The method is described in following algorithm.

\section{Algorithm 2: Key Frame Selection}

Input: series of frames

Output: Key frames

1. $k f=\emptyset$

2. $\forall I_{i=1, \ldots N}$ calculate $I(x, y)$

3. Calculate rate of change of pixel value $\nabla f=$ $\left\{\frac{d f}{d x}, 0\right\}$

4. $k f=k f \cup I$

5. end

\subsection{Face Recognition}

The face recognition module computes probabilistic values of recognizing faces that returns the index of most likely matched face from the watch list. As a consequence, we have now the list of index of faces stored in watch list that is used to apply method of computing frequent faces coming along. Evidently, face detection is prior procedure to enable any automated system to solve desire problem [26].

Suppose the detected faces are formed in a several group as follows:

$G_{1}=\left\{f_{1 j} \mid j=1,2 \ldots n_{1}\right\}$

$G_{2}=\left\{f_{2 j} \mid j=1,2 \ldots n_{2}\right\}$

$G_{3}=\left\{f_{3 j} \mid j=1,2 \ldots n_{3}\right\}$

-

$G_{n}=\left\{f_{n j} \mid j=1,2 \ldots n_{k}\right\}$

Where

$G_{i} \cap G_{j}=\varnothing$

Signifies that it is possible that person involved in a group one may be present in another groups.

\subsection{Pre-Processing}

The data required from the preprocessing task is to obtain images of moving subjects of interest and to guarantee that each separate image sequence corresponds to more than one subject only. Here we are assuming that input is provided from a video camera fixed in a constant position and continuously monitoring the scene in front of it. The groups of subjects enter in the area where they are monitored, walk towards the camera and finally exit the area. To extract faceonly from the image sequences, and normalize face tracking/extraction algorithms may be employed [26, 28, 36] (see Fig.3). These faces are the input to the next stage of the system for learning them. 

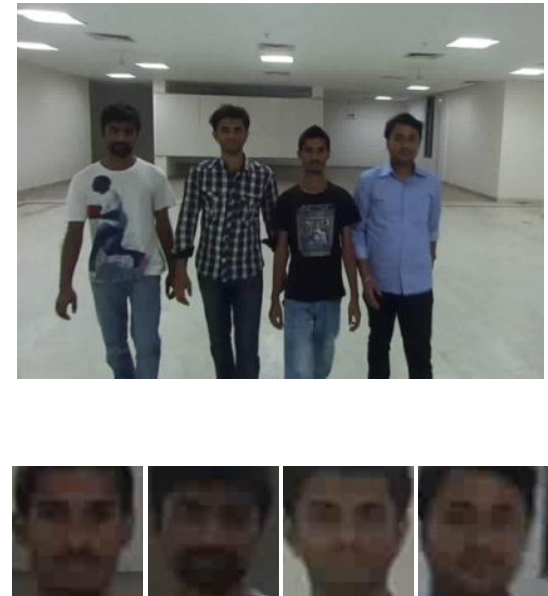

Figure 4. An example of original image and corresponding normalized 4 faces extracted from it.

\section{PATTERN MINING WITH SOCIAL NETWORK}

Frequent social network are those entities that appear in a group more frequently with some criteria specified by the system. This plays a essential roll of mining interesting pattern of social interaction and aims to analyze person's link behavior with the premise of how frequently they appear with each others.

The association rules problem was introduced by R.Agrawal et al. [22]. There is given a set of transactions, where each transaction is a set of items, an association rule is an expression of the form $X \rightarrow Y$, where $X$ and $Y$ are sets of items. The problem of discovering all association rules can be decomposed into two sub problems [AGR 94]:

First, find all sets of items that are called frequent item sets that have transaction support above minimum support.

Secondly, Use the frequent item sets to generate the desired rules.

The sub problem 1 finding frequent item sets is the essential step to discover all association rules.

Here, we are introducing the first task of association rule mining, i.e. frequent links employing the famous Apriori algorithm proposed by R. Agrawal and R. Srikant in [AGR 94]. It use BFS(Breadth First Search ) concept of finding all frequent 1-person sets, then calculating frequent 2-person sets and continues by finding subsequent larger frequent item sets.

\subsection{Problem Description}

Let us assume $\mathscr{D}$ be a set of face database. A set $F=$ $\left\{f_{1}, f_{2}, \ldots f_{m}\right\} \subseteq \mathscr{D}$ be called $\mathrm{m}$ - faceset if contains m number of faces.

In our method transaction is referred to as groups. A group over $\mathscr{D}$ is ordered pair $\mathscr{G}=($ GID, F) where GID is group identifier and $\mathrm{F}$ is faceset. A group database is $\mathrm{G}$ over $\mathscr{D}$ is set of groups over $\mathscr{D}$.

The cover of faceset $F$ contains set of group identifier of groups in $\mathrm{G}$ that support $\mathrm{F}$ :

$\operatorname{cover}(F, G)=\{$ GID $/(\mathrm{GID}, \mathrm{F}) \in \mathrm{G}, \mathrm{F} \subseteq \mathscr{D}\}$
The support of faceset $F$ in $\mathrm{G}$ is the number of groups in the cover of $F$ in $\mathrm{G}$ :

$$
\operatorname{support}(F, G)=|\operatorname{cover}(F, G)|
$$

The frequency of faceset $F$ in $G$ is the probability of $F$ appearing in group $\mathcal{G} \in \mathrm{G}$ :

$$
\operatorname{frequency}(F, G)=\operatorname{support}(F, G) /|G|
$$

A face set is frequent if its support is greater than the given absolute minimal support threshold $m s \in(0 ; 1]$.

\section{EXPERIMENTATION AND RESULT ANALYSIS}

In order to describe the experimental result of the proposed system we deploy a fixed camera in a gate to enter in our laboratory Robotics and AI lab. System captures the video sequences as per day. In this way we have a video data of several days.. The whole process of key frame generation, face detection, face recognition, and pattern mining is done offline manner to analyze the data of video. The remaining section of this experimentation will present the result from various aspect of the system.

\subsection{Key frame extraction}

First of all, we demonstrate the performance of key frame extraction video captured on the particular timing of entering the students, staff, and research scholars in the laboratory. Mostly the entities are entering and leaving constantly with making groups with different person with different time. That form of group is to be discovered as a frequent group.

While generating frames from the video several redundant frames are also generated with no information or lesser information that should be omitted or discarded

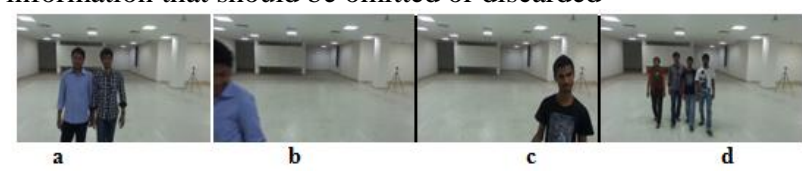

Here we observe that frame a and $\mathbf{d}$ are relevant for the context of detecting multiple faces.

\subsection{Face Detection and Recognition.}

After key frame extraction process, this module is concerned with face detection and finally recognizes it from the watch list of faces and returns the index of that recognized face. The detected faces forming 10 groups are given as in figure 4figure 15 . Here we represent only few groups for the sake of understanding.

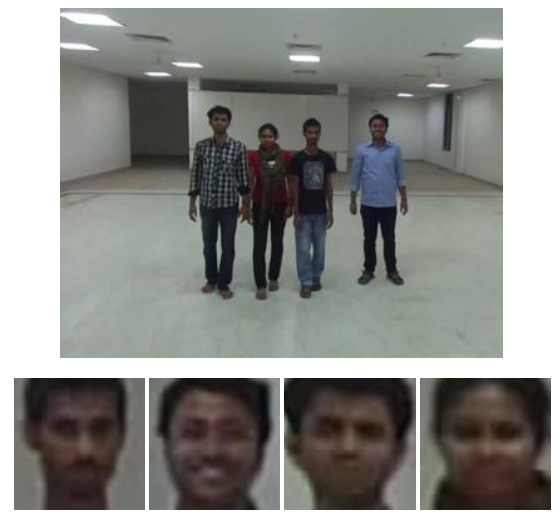

Figurg 5. Group1 and corresponding face extracted 


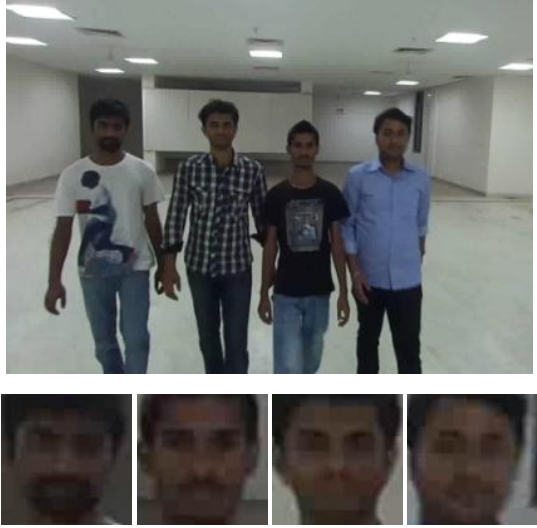

Figure 6. Group 2 and corresponding faces extracted

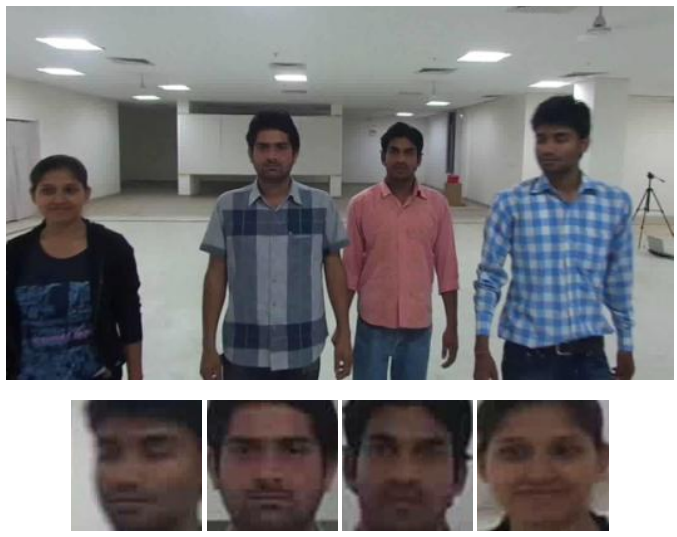

Figure 7. Group 3 and corresponding faces extracted

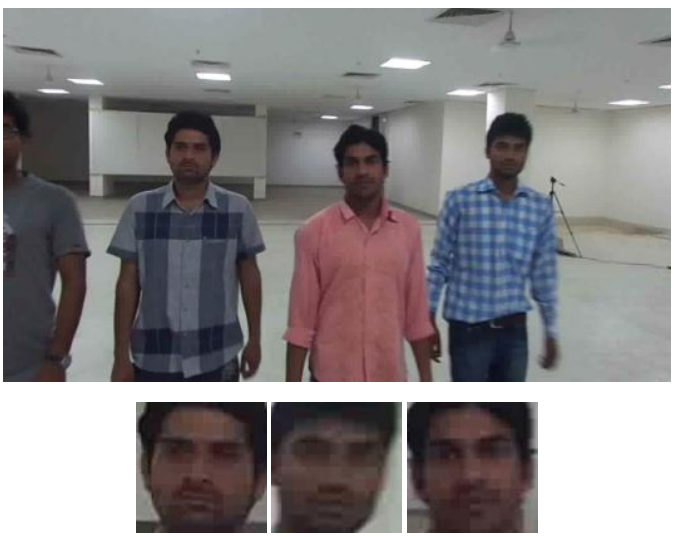

Figure 8. Group 3 and corresponding faces extracted Similarly, we have other groups with their extracted faces

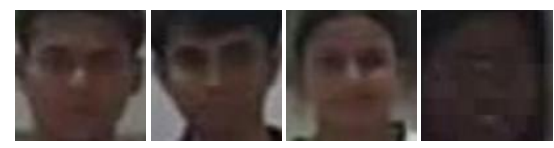

Figure 9. Group 4
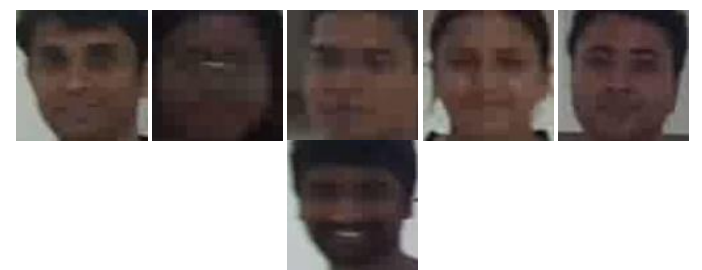

Figure 10. Group 5

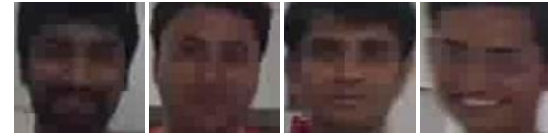

Figure 11. Group 6

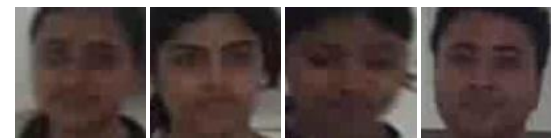

Figure 12. Group 7

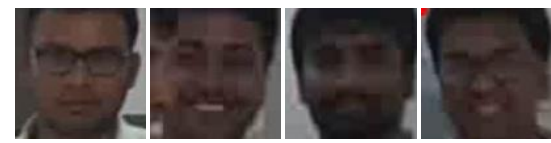

Figure 13. Group 8

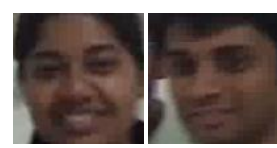

Figure 14. Group 10

\subsection{Pattern Mining with Social Network}

After detecting and recognizing faces, we are now dealing with the index of faces only that makes the transaction of group as we have item sets in general pattern mining methods. Since we have stored the face data of each person in the twelve poses that includes frontal view and several orientations of 10 degree, 20 degree, 30 degree, 40 degree and 50 degree to make our system robust that should be able to recognize faces in these variants. The final result frequent persons are given in figure 16. For the verification we have analyzed that these three persons were involved with strong link because they were associated with each other in project work.

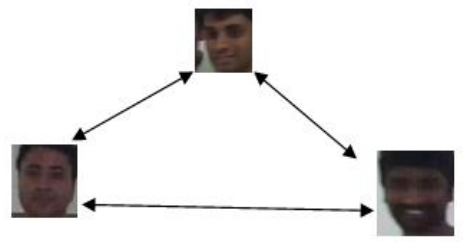

Figure 16: Social Network of frequently appearing persons

\section{CONCLUSION AND FUTURE WORK}

This paper, addressed an emerging new problem discovering and mining frequent link between social networks. A computer vision and pattern mining approach is utilized to solve the proposed problem computing frequent linked people which may have tremendous applications for practical surveillance systems. With premise of this, we have presented an algorithm to extract key frames form video sequences to identifying individuals and analyzing their interactions using face recognitions. In order to implement, a system data were collected in closed world environment using video. Several challenges were encountered to solve the problem such as to model group, face detection and recognition in cluttered environment. In a future more sophisticated and expressive strategy could be used to overcome this problem i.e. close in space with some orientation. Fuzzy concepts could be employed to clustering people to identify the degree of membership that is how strong the one associated with other 
cluster. It is also desired to extend the system with unsupervised way where dynamically-generated watch list is used in complex real world environment, whenever new faces observed during system operation are added to the watch list automatically.

\section{REFERENCES}

[1] Carley Kathleen M. 2003, Dynamic Network Analysis in Dynamic Social Network Modeling and Analysis: Workshop Summary and Papers, Ronald Breiger, Kathleen Carley, and Philippa Pattison (Eds.) Committee on Human Factors, National Research Council. Pp. 133145 .

[2] H. Schneiderman and T. Kanade. A statistical method for 3D object detection applied to faces and cars. In IEEE Computer Vision and Pattern Recognition, Hilton Head, SC, volume 1, pages 746-751, 2000.

[3] Newman, M. E. J. Detecting community structure in networks. European Physical Journal B 38: 321-330. 2004.

[4] Newman, M. E. J. Detecting community structure in networks. European Physical Journal B 38: 321-330. 2004.

[5] Newman, M. E. J Fast algorithm for detecting community structure in networks. Physical Review E 69: 066133, 2004.

[6] Luo, J. Social network analysis. Social Science Academic Press. (In Chinese), 2004.

[7] Wasserman, S. and K. Faust. Social network analysis: Methods and applications. New York, Cambridge University Press, 1994.

[8] T. Yu, S. Lim, K. Patwardhan, and N. Krahnstoever. Monitoring, recognizing and discovering social networks. In CVPR, 2009.

[9] M. E. Newman, "The structure and function of complex networks," SIAM Review, 45(2): 167-256, 2003.

[10] T. Berger-Wolf and J. Saia, "A framework for analysis of dynamic social networks," DIMACS Technical Report, vol. 28, 2005.

[11] J. Sinai, "Combating terrorism insurgency resolution software," Proc. IEEE Int. Conf. Intelligence and Security Informatics (ISI-2006), pp. 401-406.

[12] L. Backstrom, D. Huttenlocher, J. Kleinberg, and X. Lan, "Group formation in large social networks: membership, growth, and evolution," Proc. 12th ACM SIGKDD Int. Conf. Knowledge Discovery and Data Mining, 2006, pp. 44-54.

[13] D. Kempe, J. M. Kleinberg, and E. Tardos, "Influential nodes in a diffusion model for social networks." Proc. ICALP, 2005, pp. 1127-1138

[14] D. Kempe, J. Kleinberg, and E. Tardos, "Maximizing the spread of influence through a social network," in Proc. 9th ACM SIGKDD Int. Conf. Knowledge Discovery and Data Mining, 2003, pp. 137-146.

[15] Goldberg, M.; Hayvanovych, M.; Hoonlor, A.; Kelley, S.; Magdon-Ismail, M.; Mertsalov, K.; Szymanski, B.; Wallace, W.; , "Discovery, analysis and monitoring of hidden social networks and their evolution,"
Technologies for Homeland Security, 2008 IEEE Conference on , vol., no., pp.1-6, 12-13 May 2008.

[16] N. Krahnstoever, P. Tu, T. Sebastian, A. Perera, and R. Collins.Multi-view detection and tracking of travelers and luggage in masstransit environments. In In Proc. Ninth IEEE International Workshop on Performance Evaluation of Tracking and Surveillance (PETS), 2006.

[17] T. Zhao, R. Nevatia, and B. Wu. Segmentation and tracking of multiple humans in crowded environments. IEEE transactions on PAMI, 30(7):1198-1211, 2008.

[18] N. Vaswani, A. K. R. Chowdhury, and R. Chellappa Activity recognition using the dynamics of the configuration of interacting objects. In CVPR (2), pages 633-642, 2003

[19] Turaga, P., Chellappa, R., Subrahmanian, V. S., and Udrea, O. 2008. Machine recognition of human activities: A survey. IEEE Transactions on Circuits and Systems for Video Technology 18, 11 (Nov), 1473-1488.

[20] Turk, M.A.; Pentland, A.P.; , "Face recognition using eigenfaces," Computer Vision and Pattern Recognition, 1991. Proceedings CVPR '91., IEEE Computer Society Conference on , vol., no., pp.586-591, 3-6 Jun 1991.

[21] Mishra, Seema; Agrawal, Udit; Nandi, G C; , "CVPD: A tool based on a social network analysis to combating viruses propagation," Communication, Information \& Computing Technology (ICCICT), 2012 International Conference on , vol., no., pp.1-5, 19-20 Oct. 2012.

[22] R. Agrawal and R. Srikant. Fast algorithms for mining association rules. In J.B. Bocca, M. Jarke, and C. Zaniolo, editors, Proceedings 20th International Conference on Very Large Data Bases, pages 487-499. Morgan Kaufmann, 1994.

[23] R. Agrawal and R. Srikant. Fast algorithms for mining association rules. IBM Research Report RJ9839, IBM Almaden Research Center, San Jose, California, June 1994.

[24] Jiawei Han, Micheline Kember.Data Mining: Concepts and Techniques[C].Mongan Kaufmann publishers,2000.225-278.

[25] Agrawal R, Imielinski T, Swarmi A, Mining Association Rules between Sets of Items in Large Database. In: proceedings of ACMSIGMOD International conference on Management of Date Washington, D C, 1993, 207 216.

[26] M. Hegland. The apriori algorithm - a tutorial. WSPC/Lecture Notes Series, 9(7), March 2005. http://www2.ims.nus.edu.sg/preprints/2005- 29.pdf.

[27] Ming-Hsuan Yang; Kriegman, D.J.; Ahuja, N.; "Detecting faces in images: a survey," Pattern Analysis and Machine Intelligence, IEEE Transactions on , vol.24, no.1, pp.34-58, Jan 2002.

[28] Lakshmi, H.C.V.; PatilKulakarni, S.; , "Segmentation Algorithm for Multiple Face Detection for Color Images with Skin Tone Regions," Signal Acquisition and Processing, 2010. ICSAP '10. International Conference on , vol., no., pp.162-166, 9-10 Feb. 2010

[29] Seema Mishra, G C Nandi, "Hierarchy of Community in Dynamic Social Network", $2^{\text {nd }}$ International Conference 
on Soft Computing for Problem Solving SocPros 2012, 28-30 December, 2012(Inpress).

[30] J. Han, H. Cheng, D. Xin, X. Yan, "Frequent pattern mining: current status and future directions, in: Data Mining and Knowledge Discovery", $10^{\text {th }}$ Anniversary Issue, 2007, pp. 55-86.

[31] Guozhu Liu, and Junming Zhao, "Key Frame Extraction from MPEG Video Stream", Proceedings of the Second Symposium International Computer Science and Computational Technology(ISCSCT '09), Huangshan, P. R. China, 26-28,Dec. 2009, pp. 007-011.

[32] G. Ciocca and R. Schettini, "An innovative algorithm for key frame extraction in video summarization," J. RealTime Image Process., vol. 1, no. 1, pp. 69-88, 2006.

[33] R.R. Schulz and R.L. Stevenson, "Extraction of highresolution frames from video sequences," IEEE Trans. Image Processing, vol. 5, pp. 996-1011, June 1996.

[34] Rafel C. Gonzalez, Richard E Woods "Digital Image processing" Second edition Prentice Hall India.
[35] Raytchev, B.; Murase, H., "Unsupervised face recognition from image sequences based on clustering with attraction and repulsion," Computer Vision and Pattern Recognition, 2001. CVPR 2001. Proceedings of the 2001 IEEE Computer Society Conference on , vol.2, no., pp.II-25,II-30vol.2,2001

[36] Seema Mishra, G. C. Nandi; "Hierarchy of Community and Link Analysis", "Second International on Intelligent Interactive Technologies and Multimedia, Springer Communications in Computer and Information Science Volume 276, 2013, pp 246-254.

[37] A. Samal and P. A. Iyengar, Automatic recognition and analysis of human faces and facial expressions: a survey, Pattern Recognition 25, pp. 65-77, 1992.

[38] Seema Mishra, C.G. Nandi, Link Mining Using Strength of Frequent pattern of Interaction Intelligent Computing, Networking, and Informatics, Advances in Intelligent Systems and Computing 243, DOI: 10.1007/978-81-3221665-0_75. 